\title{
Los usos de los estándares de prueba: entre umbrales y prototipos ${ }^{1}$
}

\author{
The Uses of Standards of Proof: Between \\ Prototypes and Thresholds
}

Rodrigo Coloma Correa*

"Pero la certeza, generalmente, es una ilusión y el reposo no es el destino del hombre"

O. W. Holmes

La senda del Derecho

Recepción y evaluación de propuesta: 15/04/2016

Aceptación: 15/7/2016

Recepción y aceptación final: 4/7/2017

Resumen: Este texto analiza la estructura lógica y los usos de los estándares de prueba (EdP). En cuanto a lo primero, sostiene que los EdP admiten reconstruirse como umbrales o prototipos. En cuanto a lo segundo, indica que los EdP no solo se usan para distribuir falsos positivos y falsos negativos — como sostiene una posición bastante difundida-,

* Profesor de la Universidad Alberto Hurtado, doctor en Derecho por la Universidad Carlos III de Madrid, Santiago de Chile.

Correo electrónico: rcoloma@uahurtado.cl

1 Esta investigación es parte de proyecto financiado por el Fondo Nacional de Desarro1lo Científico y Tecnológico, FONDECYT 1170872, "Prueba de los hechos. Coordinación entre el lenguaje de la teoría y el lenguaje de la práctica". Este texto se ha visto beneficiado por las observaciones de varios lectores generosos que me hicieron ver distintos problemas en las versiones preliminares. En especial, agradezco los minuciosos y certeros comentarios de Daniela Accatino, Claudio Agüero y Flavia Carbonell. 
sino también, influyen en la cantidad de errores totales que produce el sistema de adjudicación, e incluso modelan la forma que adoptan los hechos probados. La operatividad de los EdP se ilustra con un ejercicio que muestra su condición tanto de prototipo como de umbral.

Palabras clave: decisiones probatorias, estándar de prueba, significados de la locución estándar

\begin{abstract}
This paper examines both the logic structure and the uses of standards of proof (SoP). On the first matter, the paper argues that SoP can be reconfigured as thresholds or prototypes. On the second matter, it states that SoP are not only used to distribute false positive and false negative type of errors - as the mainstream literature affirms-, but also can influence in the amount of total errors generated in the adjudication system, and they even can shape the way that proven facts are adopted. The functionality of SoP is illustrated with an exercise that shows their status as prototypes and thresholds.
\end{abstract}

Keywords: evidentiary decisions, standard of proof, meanings on the notion of standard

\title{
I. Introducción
}

De los problemas que aquejan a la decisión de los hechos, el concerniente a cómo deben usarse ${ }^{2}$ los estándares de prueba es de los más difíciles y, a la vez, de los que más amenazan la operatividad de los sistemas de adjudicación. En consonancia con su dimensión normativa, existen expectativas de que los estándares de prueba (en adelante, los

2 En este texto se habla recurrentemente de los «usos» de los estándares de prueba. Con ello refiero a que los estándares de prueba influyen, en distinta medida, en la elección de cursos de acción. La noción de aplicación — que indudablemente parece más familiar en el ámbito normativo - me ha parecido demasiado fuerte para los propósitos de lo que aquí presento. Para entender que un estándar de prueba ha sido usado bastará con que haya constituido una razón auxiliar para la adopción de un cierto curso de acción. 
Los usos de los estándares de prueba: entre umbrales y prototipos

$\mathrm{EdP})^{3}$ cumplan una función de guía/control sobre la toma de decisiones a cargo del adjudicador. La manera en que tradicionalmente se espera que lo hagan es por la vía de dirimir la suficiencia de las pruebas rendidas y de las argumentaciones vertidas por las partes (los EdP fijan las cargas de persuasión). En términos simples, aquello implica que si las exigencias establecidas por el EdP son superadas, los hechos debatidos en una cierta clase de procedimiento judicial debieran darse por probados; en caso contrario, no ${ }^{4}$.

Según una visión más o menos extendida, un EdP plenamente operativo ${ }^{5}$ mantiene suficientemente controlada una cierta ratio de falsos positivos y de falsos negativos. Así, los EdP resultan claves para zanjar el problema de la distribución de errores inherentes a las decisiones probatorias $^{6}$. Existe, sin embargo, la sospecha de que en la realidad la distribución de los errores en la fase probatoria no es un asunto que

3 Para no resultar fatigoso, en ciertas ocasiones con la sigla EdP estaré también refiriendo al uso de los estándares de prueba. En relación a lo último, el contexto permitirá identificar los casos en que así ocurre.

4 Los EdP fijan un parámetro de suficiencia probatoria. Si no hay claridad en ello la discrecionalidad judicial será de una amplitud tal que hará implausible sostener que el análisis de las pruebas es una tarea regulada por reglas. El problema es advertido —en términos más generales- por Allen, al indicar: "Para que el derecho probatorio pueda ser realmente regulatorio, debe normar la interacción entre lo que se aduce en el juicio y el consiguiente proceso inferencial que depende del conocimiento y de las creencias del juzgador" (Allen, R., "Los estándares de prueba y los límites del análisis jurídico”, en Vásquez, C. (ed.), Estándares de prueba y prueba científica. Ensayos de epistemología jurídica, Madrid: Marcial Pons, 2013, p. 58). En un sentido algo distinto, pero enfatizando, de todas formas, los requerimientos de precisión mínima, dice Ferrer: "La importancia de definir con claridad todos estos estándares de prueba es crucial, puesto que sin ellos no puede pretenderse una valoración racional de la prueba ni un control de la valoración realizada" (Ferrer, J., La valoración racional de la prueba, Madrid, Marcial Pons, 2007, p. 152).

5 La operatividad de los EdP supone una labor relevante de especificación previa de parte de una comunidad interpretativa. Las formas en que suelen ser formulados suelen ser, en extremo, abstractas.

6 Según Clermont, la reducción de los costos del error es una expectativa presente en los sistemas del common law. En los sistemas del civil law, en cambio, se busca proteger a la parte que no debe soportar la carga de persuasión: así, el problema que realmente le preocupa tiene más bien que ver con la legitimación de la decisión. Clermont, K., Standards of Decision in Law, Durham: Carolina Academic Press, 2013, p. 274. 
dependa, en términos fuertes, de la utilización de los EdP y, en consecuencia, de poco o nada sirven ${ }^{7}$. En lo que sigue, sugeriré que los EdP son más operativos de lo que prima facie se les hace aparecer y que el juicio adverso que sobre ellos recae es consecuencia de una visión reduccionista de sus distintos usos.

Lo que sigue se divide en dos partes. La primera parte corresponde a un ejercicio de depuración conceptual de la locución EdP. En ella se presta especial atención a lo que se quiere decir con la partícula «estándar». Según se indicará, con la palabra «estándar» se puede referir tanto a umbrales como a prototipos. La segunda parte consiste en un ejercicio de identificación de los posibles usos de aquellos umbrales o prototipos que reconocemos como EdP. Dado que son utilizados en un contexto decisional complejo, sus repercusiones no se limitan a una función puntual, sino que inciden en variadas decisiones adoptadas durante los procesos judiciales, e involucran a distintos intervinientes. Para los efectos de controlar una lectura difusa respecto de las posibilidades de uso de los EdP, se incluye en la parte final un ejercicio que muestra cómo se puede operar con ellos, tanto si se les entiende como umbrales o bien, como prototipos.

\section{1. Dos significados para la partícula «estándar»}

Del análisis del lenguaje ordinario, cabe, a lo menos, extraer dos significados para la palabra estándar:

7 En nuestro medio, las críticas de Larry Laudan — cuyo foco es el EdP del «más allá de toda duda razonable» - han ejercido una importante influencia en la actitud de sospecha que a nivel teórico se tiene de los EdP. Su principal preocupación radica en que las exigencias de los EdP se reducen a la producción de un cierto nivel de convicción en el adjudicador. Aquello, unido a la falta de precisión respecto de su significado, conspira en que se pueda hablar con propiedad de la aplicación de un EdP. Para esto último, se requeriría que los EdP fuesen objetivos y exigentes. Laudan, L., "Por qué un estándar de prueba subjetivo y ambiguo no es un estándar", Doxa, 28, 2005, especialmente p. 104 y ss. Sobre la brecha que se produce entre la teoría y la práctica en lo que concierne a los EdP, ver Dei Vecchi, D., "Tres discusiones acerca de la relación entre prueba y verdad”, Discusiones, XIII, 2013, pp. 252-260. 
Los usos de los estándares de prueba: entre umbrales y prototipos

a) Umbral que reduce $e^{8}$ los problemas extensionales de una categoría. Un acto de lenguaje constituye un estándar si define un valor mínimo (punto de quiebre) de pertenencia a una categoría ${ }^{9}$. Si en un caso concreto se iguala o supera lo que se determina en el umbra $1^{10}$, entonces todo ejemplar que antes habría sido calificado como dudoso, pasa a pertenecer a la categoría; en caso contrario, queda fuera. El ideal de un estándar, entendido como umbral, implica que el análisis que dirime si un ejemplar dudoso pertenece o no a la categoría, será puramente cuantitativo.

b) Prototipo que reduce los problemas de extensión de una categoría. La existencia de un estándar en este sentido, supone la construcción de un ejemplar que indubitadamente pertenece a una categoría y cuya función es la de resolver (algunos de) los problemas de pertenencia que afecta a casos que se sitúan en la zona de penumbra. El modus operandi de los estándares entendidos en este sentido se diferencia del precedente ya que el agente debe llevar a cabo un análisis de semejanzas y/o diferencias de los casos dudosos respecto del prototipo. La semejanza implica pertenencia y la diferencia implica no pertenencia. El análisis que se lleva a cabo cuando se entiende un estándar como prototipo es cualitativo.

8 Es importante tener en cuenta que de los estándares se espera una reducción (no una eliminación) de los problemas extensionales. En razón de ello, son admisibles formulaciones que no operen bajo una lógica binaria (sí/no) y que, por tanto, se satisfagan con la reducción de la penumbra que afecta a las categorías sobre las que se aplica. Así, se entiende que los estándares ilustren acerca de las categorías, tal como ocurre con las definiciones ostensivas.

9 La palabra categoría será utilizada con frecuencia en lo que sigue. Con ella pretendo comunicar que los significados de la partícula estándar que aquí interesan no son reducibles a escenarios puramente jurídicos. La búsqueda de estándares tiene sentido en cualquier contexto en que se aspire a alcanzar precisión o consenso en lo que debiera entenderse denotado por palabras que sirven para clasificar sujetos, objetos o fenómenos, derivándose de ello ciertas consecuencias.

${ }^{10} \mathrm{La}$ expresión «umbral» es usada por autores que refieren al EdP. Así, por ejemplo, Páez, A., "Estándares múltiples de prueba en medicina y derecho", en Páez, A. (coord.), Hechos, evidencia y estándares de prueba, Bogotá: Universidad de los Andes, 2015, p. 140 y ss. Según el Diccionario de la Lengua Española de la RAE, una de las acepciones de «umbral» es "valor mínimo de una magnitud a partir del cual se produce un efecto determinado." 
Para ilustrar lo anterior, me valdré de dos ejemplos sencillos tomados del diccionario Merriam Webster mediante los cuales se da cuenta del uso de la palabra estándar (standard), entendida esta como sustantivo (no adjetivo). Dice el aludido diccionario: a) "De acuerdo a los estándares modernos, la casa es demasiado pequeña"; b) "Este libro es el estándar respecto del cual todos los demás deberán ser juzgados". Conforme al primero de los ejemplos, de un estándar se espera la elección de una serie de variables determinantes de lo que es una casa pequeña o no pequeña (por ejemplo, metros cuadrados construidos, cantidad de piezas, etc.) y la estipulación de un nivel básico de satisfacción (por ejemplo, $60 \mathrm{~m}^{2}$ construidos, dos piezas, etc.). Conforme al segundo ejemplo, el estándar opera como un prototipo desde el cual es posible hacer comparaciones. A partir de cómo es "este libro"11 (estilo, problemática abordada, complejidad, extensión, etc.) otros libros serán calificados como «buenos», «malos» o «regulares», «novedosos», «breves», «deficientemente escritos», o con otros calificativos por el estilo.

En los párrafos sucesivos, se demostrará que ambos significados son útiles para explicar los EdP.

\section{2. ¿Qué implica la partícula «estándar» en los contextos jurídicos?}

La discusión acerca de lo que se quiere decir con «estándares» es escasa. Aquello se explica por la circunstancia de que hasta hace algunos años, resultaba una expresión casi ausente en los discursos de jueces y abogados del civil law. La escasez de discusión - y, en consecuencia, de teoría- incide en que las diferencias que separan los estándares del resto de las directivas jurídicas son poco nítidas. ${ }^{12}$ A continuación indicaré

${ }^{11}$ El prototipo puede expresarse mediante la identificación de un ejemplar concreto que pertenece a la categoría (como ocurre cuando se señala un libro concreto para aludir a la categoría de los libros deseados), pero también mediante el señalamiento de un ejemplar ideal como lo sería cuando se alude al "hombre medio".

${ }^{12}$ La irrupción de los estándares de prueba en el civil law — podría pensarse - se debe fundamentalmente a la influencia que ha sido ejercida por el common law en el que, a diferencia nuestra, sí se contaba con una larga tradición en su uso. Sobre esto último, ver Clermont, K., op. cit., p. 256-257. 
Los usos de los estándares de prueba: entre umbrales y prototipos

algunas de las características más sobresalientes del uso de estándares en el mundo del derecho:

a) El uso de estándares se produce (normalmente) en contextos en que uno o más agentes deben adoptar decisiones justificadas. Los estándares sirven para dirimir conflictos acerca de si un caso cualquiera forma o no parte de una categoría a la que, por anticipado, se vincula una determinada reacción. De los estándares se espera, por una parte, la eliminación (o reducción al mínimo) de potenciales respuestas que no calcen con estructuras de comunicación propias de una lógica binaria ${ }^{13}$, esto es, en que solo se opera con las opciones: «este es un caso de $X$ » $\mathrm{y}$ «este no es un caso de $X »^{14}$. La lógica binaria excluye matices que en otros escenarios son fundamentales para enriquecer las explicaciones. Aquella merma se entiende compensada por su contribución a la disposición oportuna de respuestas para enfrentar problemas prácticos.

En contextos en que un agente no se vea forzado a tomar decisiones sino en que simplemente se espera que exteriorice sus creencias, no resulta incómodo, ni tampoco desaconsejable el uso de debilitadores discursivos como «quizás», «probablemente» $\mathrm{u}$ otros similares. El uso de estos permite introducir matices a las muy reducidas opciones ofrecidas por los códigos binarios $^{15}$. Al contrario, en contextos que podrían llamarse

${ }^{13}$ Los estándares excluyen respuestas que, en una importante medida, propenden a que las discusiones respecto a la pertenencia a una categoría permanezcan abiertas, como ocurre cuando se recurre a expresiones tales como, "probablemente» o «quizás». La eliminación de los matices es objeto de crítica. La lógica borrosa se ha propuesto como una forma alternativa de dar cuenta del razonamiento que asuma su carácter "aproximado", antes que preciso. Zadeh, L., "Fuzzy logic and approximate reasoning", Synthese 30, 1975, p. 407.

${ }^{14} \mathrm{La}$ aparente inevitabilidad de la lógica binaria en el campo jurídico se deriva, en gran medida, de que no se está dispuesto a graduar las penas o las indemnizaciones dependiendo del valor que sea asignado a la masa de prueba disponible.

${ }^{15}$ Los códigos binarios imponen un mayor nivel de responsabilidad sobre el agente que los usa. Al moverse solo entre opciones sí/no, se ponen en juego las competencias de razonamiento del agente. Al contrario, aun cuando no se acierte en un juicio, si se matizó con la expresión "probablemente», las eventuales críticas se suavizan. Ver Austin, J. L., “Otras mentes”, en Urmson, J. O. y Warnock, G. J. (coomp.), Ensayos filosóficos, Madrid, Alianza, 1989, p. 103-108. Toulmin, S., Los usos de la argumentación, 
decisionales (y también, en contextos tecnológicos), es indispensable simplificar la realidad por la vía de declarar relevantes algunos asuntos e irrelevantes otros.

Por otra parte, los estándares son usados bajo el entendido de que la decisión que se tome habrá de ser justificada. En ese sentido, la clausura a la que propenden es decisional y no discursiva. La aplicación del estándar no inhibe, entonces, la justificación del curso de acción adoptado.

b) La utilidad de los estándares concierne a asuntos que prima facie se sitúan en la zona de penumbra de una categoría. Resultan, entonces, innecesarios respecto de asuntos que tempranamente admitan ubicarse en las zonas de claridad o de obscuridad de la categoría que interesa. En estos últimos casos, basta con tener en cuenta las propiedades definitorias de la respectiva categoría.

Ante el abandono total de un encargo por parte de un mandatario - sin que se cuente con un sustituto y habiéndose derivado graves perjuicios por su falta de atención- la remisión a un estándar de diligencia, como lo es el del «buen padre de familia», no pasa de constituir una mera formalidad. Las propiedades definitorias de diligencia resultan más que suficientes para resolver el problema en términos de que «el agente ha sido negligente».

Para los efectos de hacer desaparecer (o, al menos, reducir) la penumbra en una categoría es necesario simplificar los problemas de indeterminación que aquejan la tarea interpretativa. Aquello supone declarar irrelevantes algunas de las posibilidades de uso que ofrece la categoría que interesa y/o estipular niveles de logro en otras.

Barcelona, Península, 2007, pp. 73-79. Respecto de la importancia del estándar de prueba "más allá de toda duda razonable" a los efectos de disminuir la angustia moral que históricamente se producía en los juzgadores que debían tomar decisiones de condena en contextos de incertidumbre, ver Whitman, J., The origins of reasonable doubt. Theological roots of the criminal trial, New Haven \& London: Yale University Press, 2008, p. 10 y ss. 
Los usos de los estándares de prueba: entre umbrales y prototipos

El problema de la responsabilidad del constructor que se encuentra obligado a operar conforme a ciertas expectativas de diligencia se zanja — por ejemplo- mediante la revisión de si cumplió o no con los estándares exigidos para la construcción de viviendas. Los estándares sustituyen el análisis de la diligencia a partir de las propiedades que ordinariamente la definen.

c) Los estándares requieren conservar un nivel razonable de la complejidad de cada caso que se evalúa. Para ello, los estándares toman en cuenta simultáneamente varios criterios que dirimen la pertenencia o no pertenencia a una categoría. Los objetos o conductas sometidas a evaluación pueden resultar muy satisfactorios en una cierta dimensión y escasamente en otras, lo que es relevante a los efectos de decidir su pertenencia a la categoría que interesa ${ }^{16}$.

La decisión de si un automóvil será considerado como un «automóvil seguro» debe tener en consideración variables tales como su capacidad de frenado, el nivel de deformación que se produce ante impactos a distintas velocidades, la performance alcanzada en situaciones de conducción anormales como ocurre cuando hay nieve en las calles, el nivel de iluminación de sus focos, etcétera. Un estándar bien formulado admite simplificarse (menos variables), bajo la condición de que no haya pérdida en ello.

d) Los estándares consideran variables contextuales. La diferente manera de formular e interpretar un estándar depende de las posibilidades que ofrezca el entorno (por ejemplo, tecnología disponible) y de las posibilidades de producción de prueba que tengan los sujetos concretos ${ }^{17}$.

16 Esta expectativa sobre los estándares enfatiza su diferencia respecto de las reglas. En lo que atañe a estas últimas, se opta por simplificar las categorías relevantes para el otorgamiento de beneficios o imposición de cargas. Ver Hart, H. L. A., El concepto de derecho, Buenos Aires: Abeledo-Perrot, 1995 (2. ${ }^{a}$ reimpresión de la 2. ${ }^{a}$ edición), pp. 155-159. En el sentido indicado, los estándares propenden a que se opere desde un subsistema normativo en que es posible identificar varios casos.

17 A propósito de los «estándares de coherencia» de la justificación jurídica, Amalia Amaya destaca la variación que experimentan según el contexto en que se apliquen. 
Aquello da lugar a que se hable de estándares modernos o tradicionales, de estándares exigentes o poco exigentes (relajados). Los estándares exigentes son especialmente útiles si se quieren evitar los riesgos de que se cuele un ejemplar indeseable en una categoría respecto de la que se discute. A la vez, son problemáticos por los altos costes en que debe incurrirse para satisfacerlos y porque dejan fuera ejemplares dudosos ubicados cerca de la zona de claridad. Su problema es la infrainclusión. Los estándares no exigentes, como contrapartida, son ineficientes para eliminar ejemplares que no cuentan con suficientes credenciales de pertenencia a la categoría y, a la vez, no generan incentivos para mejorar procesos de producción (sin necesidad de esfuerzo es muy probable que los ejemplares dudosos se acepten en la categoría). Su problema es la sobreinclusión.

Lo señalado incide, por ejemplo, en que la calificación de determinadas conductas como «diligentes» (o no) variará según si el estándar es el del «buen padre de familia» 0 «de las personas negligentes y de poca prudencia». Lo mismo es lo que lleva a que el carácter de probado o no probado de una cierta conducta varíe según si el estándar aplicado es el de la justicia penal o de la civil.

\section{3. ¿Qué es un estándar de prueba?}

La decisión de dar por probado (o por no probado) un hecho en contextos judiciales supone la coordinación entre un contenido (lo afirmado), una actitud (aceptación o rechazo), y un conjunto de razones que sirven

Como variables determinantes del contexto refiere, entre otras, a la importancia de las consecuencias, a las restricciones metodológicas, a los recursos disponibles e, incluso, a rasgos dialécticos. Amaya, A., "Diez tesis acerca de la coherencia en el derecho" Discusiones X, 2011, pp. 38-43. Respecto de los EdP, hay buenas razones para considerar las posibilidades que las partes del juicio tienen de producir prueba. Ver Larroucau, J., "Hacia un estándar de prueba civil", Revista Chilena de Derecho, 39 (3), p. 797 y ss.; Coloma, R., "Estándares de prueba y juicios por violaciones a los derechos humanos", Revista de Derecho (Valdivia), XXII (2), 2009, p. 225 y ss. 
Los usos de los estándares de prueba: entre umbrales y prototipos

de soporte a la actitud que se adopta frente al contenido en cuestión ${ }^{18}$. En otras palabras, en sistemas jurídicos como los nuestros, para lograr que la conjetura formulada en la fase inicial del proceso adquiera el estatus de probado o no probado, no basta la actitud de aceptación o de rechazo, respectivamente. Se requiere también de la construcción de una narrativa que se haga cargo de la prueba rendida y de los argumentos vertidos por las partes, en términos consistentes con lo decidido.

Los contenidos posibles de un hecho probado/no probado se encuentran más o menos limitados por lo estipulado en la condición de aplicación de una o más normas jurídicas. Son las partes en un juicio las que - por regla general — soportan la carga de alegar una determinada forma de vinculación entre un pretendido episodio de la vida y aquello prescrito en el sistema jurídico. La discusión resulta más fructífera en la medida en que - en una fase temprana del procesose fije una conjetura definitoria de los hechos a probar. Quedarán, así, delimitados los contornos de la fase del proceso judicial conducente tanto a producir pruebas como, argumentaciones (¿de qué vamos a discutir en sede probatoria?). Para construir esa conjetura se requiere un esfuerzo interpretativo importante acerca de las relaciones posibles entre las disposiciones y los relatos ofrecidos por las partes. Al momento de definir el contenido de los hechos que se espera sean probados, los EdP no parecieran jugar un rol relevante.

Los EdP juegan un rol clave y directo para la adopción de actitudes. La manera en que operan es dirimiendo si los casos dudosos deben o no ser situados en la categoría de hechos probados en un proceso judicial. Dicho en otras palabras, los EdP contribuyen a la toma de decisiones en todos aquellos casos que se sitúan en la zona de penumbra de la conjetura. Los EdP, además, hacen posible que ante la misma conjetura - pero de la que se derivarán soluciones distintas (una pena, una indemnización)— se puedan tomar decisiones diferentes ${ }^{19}$. Así las cosas, los

${ }^{18}$ Lo expresado da cuenta de lo que ocurre con cualquier aserción que aspire a ser considerada como justificada. Pepper, S., World Hypotheses, Berkeley: University of California Press (impresión según demanda, año publicación 1942), pp. 11 y 12.

${ }^{19}$ La dependencia del contexto en lo que concierne a lo que llegarán a ser nuestras actitudes no es, por cierto, un asunto que se produce solo en el espacio de lo jurídico. 
EdP deben dar indicaciones útiles acerca de las condiciones de las cuales se hará depender la actitud «hecho $p$ probado: sí»; o su opuesta, «hecho $p$ probado: no». Los EdP son construidos, entonces, con la pretensión de que operen como reglas determinativas o definitorias.

Las razones que pueden esgrimirse para calificar los hechos como probados o no probados son muy variadas. Las pruebas disponibles admiten múltiples formas de valoración y, por tanto, las razones dependen, en una importante medida, de lo que plantee cada una de las partes. Por cierto, hay razones más atendibles que otras. Las posibilidades de uso de los EdP en lo que concierne a las razones son indirectas. En otras palabras, los EdP no determinan las razones a esgrimir en un caso concreto, pero sí orientan a las partes respecto de si vale o no la pena invocar un conjunto de razones y no otro, según la actitud que se espera provocar en los juzgadores ${ }^{20}$. Se recurre para ello a las posibilidades que ofrecen los umbrales o prototipos.

Las formulaciones más consolidadas de los EdP son las de: «preponderancia de la prueba» $(\mathrm{PP})$, prueba «clara y convincente» $(\mathrm{CC}) \mathrm{y}$ «más allá de toda duda razonable» (MADR), siendo imaginables, por cierto, algunas otras. En principio, podría entenderse que la PP busca determinar si los hechos discutidos en un proceso judicial admiten calificarse como hechos probados, por la vía de ubicarlos en una cierta posición dentro de una escala de grados de prueba (aproximación a un umbral). En el caso del CC, la pertenencia a una categoría (de los hechos probados) pareciera lograrse por la vía de un juicio de semejanzas (aproximación a un prototipo). E1 MADR es más discutible ya que admite una lectura en ambas direcciones ${ }^{21}$.

Ver, por ejemplo, Nozick, R., The nature of the rationality, tercera edición, Princeton: Princeton University Press, 1995, p. 98.

${ }^{20}$ Esto también refiere a las razones que los jueces darán a los lectores de sus sentencias. Así, la argumentación de las sentencias está determinada, en parte, por lo que exigen los EdP. Todo esto se desarrolla a continuación al referirse a los usos de los EdP.

${ }^{21}$ Larry Laudan sostiene que es usual hallar dos clases de aproximación respecto del MADR. Por una parte, los tribunales de justicia suelen privilegiar una aproximación cualitativa (la preocupación, es por ejemplo, la clase de convicción que produce la prueba rendida) y, por la otra, los académicos están preocupados de fijar un umbral de probabilidad (bayesiana) que debe alcanzar la parte sobre la que recae la carga 
Los usos de los estándares de prueba: entre umbrales y prototipos

Entendidos como umbrales, los EdP suponen la posibilidad de construir escalas de medición. Así, las interpretaciones buscan -a través de cifras o conceptos- reducir las variables relevantes del caso y, de esa manera, provocar consensos metodológicos. El cálculo probabilístico, pese a todas sus dificultades, resulta prometedor para esta perspectiva. Entendidos como prototipos, los EdP alientan las comparaciones con un caso cuya pertenencia a la categoría «hecho probado» no resulta dudosa. Así, por ejemplo, podría entenderse que a través del CC se busca representar un tipo de justificación que implica el uso de una narrativa que no abusa de distinciones ni del lenguaje rebuscado (clara) y, a la vez, que es apta para provocar en el interlocutor adhesión respecto de la estructura argumental utilizada (convincente) ${ }^{22}$. La inferencia de la mejor explicación podría funcionar bien en esta sede.

de la prueba (Laudan, L., "Por qué un estándar de prueba subjetivo y ambiguo no es un estándar", Doxa, 28, 2005, pp. 98-99. Ver también Laudan, L., Verdad, error y proceso penal, Madrid: Marcial Pons, 2013, pp. 66-83). Aun cuando lo que preocupa a Laudan es la subjetividad y ambigüedad que puede detectarse en ambas aproximaciones redundando en que - a su juicio - el MADR no sea un EdP genuino, la distinción referida es ilustrativa de que el MADR admite ser reconstruido tanto como un prototipo (debes estar tan convencido como en aquellos casos en que...), o bien como umbral (habiéndose probado un $90 \%$, entonces...).

Las fronteras son difíciles de delinear. Ferrer pareciera simpatizar con la concepción de los EdP como umbrales. Así, él sugiere que "es imprescindible fijar el umbral a partir del cual aceptaremos una hipótesis como probada" aclarando a continuación que no es posible una "cuantificación numérica". Sin embargo, cuando hace una propuesta de cómo usar el MADR señala que: "1) La hipótesis debe ser capaz de explicar los datos disponibles, integrándolos de forma coherente, y las predicciones de nuevos datos que la hipótesis permita formular deben haber resultado confirmadas..." aparece mucho más comprometido con una perspectiva propia de un prototipo. (Ferrer, J., op. cit., p. 139, 147).

${ }^{22}$ A diferencia de lo que ocurre con el ejemplo del libro, el prototipo aquí referido tiene un alto nivel de abstracción. En rigor, un prototipo muy abstracto presenta problemas importantes para su identificación pues da amplio espacio al desacuerdo en la comunidad de intérpretes. 


\section{II. ¿Cuáles son los usos de los estándares de prueba?}

Los EdP admiten usos variados: algunos de ellos son directos y otros, indirectos. En lo que sigue se considerará, especialmente, lo que ocurre en el civil law, sin perjuicio de que la mayoría de los usos que se sugieren se aplican también en el common law $^{23}$. Los EdP adquieren protagonismo no solo en la fase de la decisión probatoria, sino también en las etapas previas y posteriores de un juicio. La razón es aparentemente de Perogrullo: tanto las partes como el tribunal adaptan sus actuaciones según el impacto que, estiman, llegarán a tener en la decisión que da o no por probadas las acciones $u$ omisiones ${ }^{24}$ sobre las que se discute. La enumeración de los usos de los EdP que a continuación se hace no es exhaustiva ${ }^{25}$ (no se agotan sus distintas posibilidades) y, a la vez, en varios de los casos presentados tiene un carácter meramente exploratorio (algunos de las propuestas podrían reformularse o fusionarse):

${ }^{23}$ Las diferencias obedecen, principalmente, a que en el civil law la decisión probatoria corresponde a jueces profesionales, y a que esta debe motivarse (Taruffo, M., "Tres observaciones sobre 'Por qué un estándar de prueba subjetivo y ambiguo no es un estándar' de Larry Laudan", Doxa, 28, 2005, pp. 119-122). Según Laudan, ni la participación de jueces profesionales, ni la obligación de motivar cambia mayormente la situación respecto al MADR (Laudan, L., "Una breve réplica”, Doxa, 28, 2005, pp. 152-155). Al observar el comportamiento de los tribunales, Laudan pareciera llevar la razón. Hay, sin embargo, algunas diferencias que es útil no diluir respecto del papel de los EdP. En el listado que se propone en este trabajo, habrá que prestar atención a las letras c) y f).

${ }^{24}$ En rigor, también se dan por probados estados de cosas, sobre todo, en casos en los que se persigue la responsabilidad objetiva de un sujeto. El foco en la prueba de acciones $\mathrm{u}$ omisiones se explica dado que a nivel de uso de EdP se pone mayor atención en el probandum final que en los intermedios. Anderson, T., Schum, D. y Twining, W., Análisis de la prueba, Madrid: Marcial Pons, 2015, p. 409.

${ }^{25}$ Un análisis interesante que amplía las posibilidades de uso de los EdP — tal como aquí se promueve - puede verse en Ho, H. L., A Philosophy of Evidence Law, Oxford: Oxford University Press, 2010 (reimpresión), p. 185 y ss. Ho llama la atención acerca de la conveniencia de adoptar una forma de análisis de los EdP, desde una perspectiva interna. Aquello implica considerar su dimensión en cuanto estándares de precaución, es decir de una actitud de resistencia a ser persuadido de la verdad de la conjetura de la que judicialmente se discute. Lo expresado redunda en que en el EdP de la PP (aplicable para materias civiles) promueva la actitud de imparcialidad del juzgador; en cambio, el EdP del MADR (aplicable en el ámbito penal,) lo que promueve es una actitud de protección en favor del acusado. 
Los usos de los estándares de prueba: entre umbrales y prototipos

a) Orientan a las partes respecto de la conveniencia de participar o de retirarse anticipadamente en un litigio. Por una parte, los EdP dan señales al demandante o al acusador acerca de cuándo conviene iniciar un proceso judicial y, a la vez, de cuándo es preferible abstenerse de hacerlo. Por la otra, los EdP estimulan o desincentivan a la demandada o acusada a transigir, a perseverar o a rendirse tempranamente. Acorde a lo indicado, un estándar poco exigente estimula la interposición de demandas y, a la vez, hace aconsejable a la parte demandada transigir para salvar parte de lo disputado, e incluso, reconocer sus responsabilidades para ahorrarse los costes de la litigación. Al contrario, un estándar altamente exigente desincentiva la interposición de demandas, insta a los demandantes a estar más llanos a negociar en caso de desacuerdo, como también, da señales a los demandantes en orden a no ser propensos a aceptar transacciones ${ }^{26}$.

b) Orientan a las partes acerca de cuánta prueba conviene producir en un proceso judicial. Un buen manejo de los EdP disminuye los problemas de hiper racionalidad (producción y/o análisis de prueba sobreabundante) o de irracionalidad (producción y/o análisis de prueba escasa) ${ }^{27}$. Si las demás variables se mantienen constantes, un EdP altamente exigente implica que el demandante deberá asumir elevados costos para salir victorioso y, al contrario, que la demandada no debiese invertir demasiados recursos en la producción de pruebas, ni en la construcción de argumentos. A su vez, un EdP poco exigente implica para el demandante costes reducidos de producción de pruebas y de argumentos. A la vez, para la demandada implica un esfuerzo equiparable al de este, si no quiere ser derrotada.

En la realidad, las otras variables relevantes que inciden en la cantidad de prueba a producir no permanecen inalteradas. Un EdP altamente exigente se asocia a decisiones que impactan de manera importante en la vida del acusado o demandado, por lo que este no se

${ }^{26}$ Ver Allen, R., op. cit., pp. 49-50; Anderson, T., Schum, D. y Twining, W., Análisis de la prueba, Madrid: Marcial Pons, 2015, p. 284 y ss

${ }^{27}$ Elster, J., Juicios salomónicos. Las limitaciones de la racionalidad como principio de decisión, Barcelona: Gedisa, 1991, p. 19. 
arriesgará a producir poca prueba. La parte acusadora o demandante, que sabe aquello, se preocupará de producir aún mejores pruebas y argumentos si no quiere fracasar en su intento probatorio. Un EdP poco exigente no lleva necesariamente a una producción moderada o baja de pruebas y argumentos: la cuantía de lo que se encuentra en juego podría implicar un gran esfuerzo de ambas partes conducente a una producción alta de pruebas y argumentos.

c) Modelan la clase de inferencias que es válido realizar desde las pruebas disponibles hacia las conjeturas a probar. Los EdP definen la magnitud de los saltos argumentales que resultan tolerables, ya sea en los alegatos de las partes o en el texto de las sentencias ${ }^{28}$. Los saltos intolerables - podría decirse - son aquellos que van mucho más allá de lo que sus premisas permiten. Por cierto, las premisas en sí no fijan sus alcances, sino la respectiva comunidad de intérpretes. Los EdP asumen dicha tarea por la vía de indicar autoritativamente cuán arriesgadas (o conservadoras) se espera que sean las inferencias probatorias que se proponen, tanto en los alegatos de los abogados como en las sentencias de los jueces ${ }^{29}$.

Un EdP exigente es escasamente tolerante a la realización de inferencias arriesgadas a partir de la prueba disponible (no hay espacios para realizar saltos argumentales muy amplios). Un EdP poco exigente, en cambio, está abierto a la inclusión de inferencias que no eliminen explicaciones alternativas plausibles (las cadenas de argumentos admiten una mayor separación entre sus eslabones). Conforme a lo indicado, el texto de una sentencia condenatoria en casos que corres-

${ }^{28}$ En términos generales, los EdP se usan para evaluar la legitimidad de una red de argumentos completa, más que de distintos tramos de argumentos. Dado que las inferencias representan múltiples conexiones entre los eslabones que forman parte de la red argumental, podría pensarse que los EdP no actúan a un nivel micro como el que se sugiere. Esta objeción no vendría al caso. La red argumental puede perder mucha fuerza por solo una inferencia dudosa (como no es de extrañar que ocurra con la duda razonable). A ello se suma la circunstancia de que los EdP orientan a los participantes acerca de la rigurosidad con la que deben operar en cada paso argumental que realicen si se asume una perspectiva más atomista que holística (piénsese, por ejemplo, en el chart method de Wigmore).

29 Coloma, R.; Agüero, C., "Lógica, ciencia y experiencia en la valoración de la prueba", Revista Chilena de Derecho, vol. 41, N², 2014, pp. $681-685$. 
Los usos de los estándares de prueba: entre umbrales y prototipos

ponda aplicar un EdP altamente exigente debiese ser exhaustivo en orden a reducir, en la mayor medida de lo posible, ya sea las lagunas explicativas, o bien las contradicciones entre las distintas partes del discurso. Especial cuidado deberá tenerse en la eliminación de explicaciones alternativas que se encuentren dotadas de plausibilidad y que apunten en una dirección opuesta a la elegida. El texto de la sentencia condenatoria en que se aplique un EdP poco exigente puede no ocuparse de destruir cualquier explicación alternativa, salvo que exista prueba que fortalezca alguna de tales explicaciones alternativas. Al contrario, en sentencias absolutorias en que se aplique un estándar exigente podrá bastar con mostrar cualquier fisura argumental que haga plausible una explicación diferente a la sostenida por la parte demandante o acusadora. Por su parte, en sentencias absolutorias en que se aplique un estándar poco exigente habrá que preocuparse por dejar asentadas lagunas o contradicciones importantes en las explicaciones de la demandante o acusadora; ello en términos de que el curso alternativo que ofrece aparezca como tan plausible como el que se desecha.

d) Distribuyen riesgos de falsos positivos y falsos negativos. La concepción hegemónica de los usos de los EdP se focaliza en el problema de la distribución de los falsos positivos y de los falsos negativos. Se trata de una versión fuerte, ya que de lo que aparentemente se hace cargo es de la zona de discrepancia habida entre lo que se da o no se da por probado, por una parte, y aquello que realmente ocurrió en el mundo, por la otra ${ }^{30}$.

Según la formulación recién anunciada, este uso de los EdP se anticipa como inservible pues - como es de sobra sabido - no nos encontramos en la posición del "Ojo de Dios" respecto de lo que realmente $e^{31}$ ha ocurrido en el mundo y, por tanto, la referida comparación parece

${ }^{30}$ La crítica de Juan Carlos Bayón en cuanto a que los EdP se limitan a establecer relaciones ordinales, siendo impotentes, en cambio, para comparaciones cardinales, es una observación que da cuenta de lleno de los problemas que aquejan a la versión fuerte de la distribución de falsos positivos y falsos negativos. Bayón, J. C., "Epistemología, moral y prueba de los hechos: hacia un enfoque no benthamiano", Analisi e Diritto, 2008, p. 31, 32.

${ }^{31}$ La locución "ocurrió realmente" es extraña. Aparece como una tautología, pero si analizamos cómo usamos el lenguaje no lo es. En una cierta medida, de lo que se trata es de fijar una posición de poder. 
imposible de realizar. Una posible salida pasa por resignificar los términos «falso positivo» $\mathrm{y}$ «falso negativo» (versión débil). De lo que se trata es de exigir una determinada posición epistémica ${ }^{32}$ (más/menos fuerte), ya sea en el momento de la decisión o posteriormente ${ }^{33}$. Así, por ejemplo, si habiéndose ya condenado a los acusados por un delito de homicidio aparece viva la presunta víctima, nos encontraremos en inmejorables condiciones para sostener que en la sentencia se incurrió en un falso positivo. Eso no es todo, también estaremos en una confortable posición epistémica para alegar un falso positivo, si con posterioridad a la condena se aprehende a otros sujetos respecto de los cuales se genera prueba mucho más apabullante acerca de su culpabilidad que la que, en su momento, estuvo disponible respecto de quienes fueron condenados.

Un EdP escasamente exigente establece un contexto propicio para el aumento de los falsos positivos (se dan por probados hechos respecto de los cuales las pruebas de cargo no han sido especialmente fuertes y, por tanto, no sería impensable que en el futuro se lleguen a producir pruebas que las desvirtúen), en cambio, un estándar altamente exigente establece un contexto en que no resultan infrecuentes los falsos negativos (no se dan por probados hechos respecto de los cuales las pruebas son fuertes, siendo previsible que en el futuro no sean desvirtuadas $\mathrm{e}$, incluso, resulten fortalecidas). El primer escenario beneficia a los demandantes y el segundo, a los demandados.

e) Determinan los opuestos que compiten en la decisión probatoria. Las opciones en competición pueden ser: a) «probada la conducta $X$ » v/s «no probada la conducta $X » ;$ o b) «probada la conducta $X$ » v/s «probada la conducta no $X »$. En el primero de los casos el opuesto a la decisión de dar por probada la conducta $X$ es la suspensión de la deci-

32 Susan Haack habla a propósito de los EdP en términos de grados de aval, lo que es consistente con la versión débil aquí presentada. Haack, S., "El probabilismo jurídico: una disensión epistemológica”, en Vásquez, Carmen (ed.), Estándares de prueba y prueba científica. Ensayos de epistemología jurídica, Madrid: Marcial Pons, 2013, pp. 75-89.

${ }^{33}$ Si se asume una versión débil de la distribución de falsos positivos y falsos negativos, la satisfacción de la expectativa de establecer relaciones ordinales entre los cursos de acción no constituye un mal resultado. Aquello, por lo demás calza bastante bien con la concepción de los EdP como prototipos. 
Los usos de los estándares de prueba: entre umbrales y prototipos

sión dar por probado $X$ (negación ilocucionaria). En el segundo de los casos, el opuesto a la decisión de dar los hechos por probados $(X)$ es la decisión de dar por probada la conducta opuesta, es decir no $X$ (negación locucionaria o proposicional). $\mathrm{El}$ asunto, por cierto, es relevante respecto de la forma en que se llevará a cabo el juicio ${ }^{34}$. La primera perspectiva pareciera ser la propia del MADR; en cambio, la segunda es la que mejor representa a la PP.

$\mathrm{Al}$ no considerarse la lógica propia de una competición entre puntos de vista contrarios, sino solo una determinada forma de dar cuenta de la realidad (por ejemplo: Rodión mató a Aliona), en el caso del MADR la función de la defensa se reduce a debilitar las razones por las cuales habría que aceptar dicha reconstrucción. En el caso de la PP compiten dos reconstrucciones de un evento: Montgomery pagó el precio pactado / Montgomery no pagó el precio pactado. En vista de ello, la parte sobre la que no recae el EdP debe preocuparse no solo de desvirtuar lo afirmado por la contraparte, sino también de suscitar adhesión a la versión que defiende ${ }^{35}$.

f) Influyen en la clase de razonamiento que las partes y los jueces adoptan para conectar la prueba disponible y la conjetura que se somete a contrastación. Los enfoques que compiten son, por una parte, de carácter atomista, lo que implica que el analista divida la conjetura en diferentes proposiciones (penúltimas) a demostrar. Cada una admite un análisis inde-

${ }^{34}$ La forma de participar en el juicio cambia considerablemente según los opuestos que compitan. En el primero de los casos presentados, a la parte sobre la que no recae la carga de la prueba le basta con debilitar la versión de la contraria en términos que no llegue a ser aceptada (actúa como un aguafiestas: solo debe destruir); en el segundo de los casos, la parte que no soporta la carga de la prueba debe debilitar el punto de vista rival y, a la vez, construir un relato alternativo.

${ }^{35}$ Como hipótesis, cabría sostener que la PP admite reconstruirse desde la perspectiva del demandado, sin que ello provoque alteraciones en los procesos en que esta se aplica. Una fórmula podría ser la de que la demandada debe soportar un EdP de «al menos una prueba que resulte equiparable a la que presente el demandante». En la práctica sería difícil que funcione algo así, ya que la $\mathrm{PP}$ suele entenderse asociada a la exigencia de que la parte que debe soportar el EdP tiene la obligación de presentar un nivel de prueba que haga al menos plausible lo que sostiene. Ver Schauer, F., Profiles, Probabilities and Stereotypes, Cambridge \& London: The Belknap Press of Harvard University Press, 2006, p. 79 y ss. 
pendiente. Cuando aquello ocurre, la cadena argumental resulta tan delgada como lo sea su eslabón más delgado. En el MARD al excluirse del estatus de probado las conjeturas respecto de las cuales se entiende que persiste una duda razonable, se favorece el desglose del análisis en tantas variables como resulte útil a la defensa a los efectos de identificar una duda razonable.

El razonamiento holístico se ocupa de abordar el problema como un todo. No importa la debilidad de algunas variables, sino lo que ocurre con el conjunto. Así, el caso no se analiza como un diagrama argumental en que se desglosan las diferentes variables, sino como una red en la que algunas partes débiles son sostenidas por las más fuertes. En la PP, en cambio, se favorecen formas de representación argumental que dejan de lado cuestiones de detalles, y el foco se dirige hacia la comparación de los distintos relatos en competición.

g) Inciden en la elección entre una concepción de los hechos probados que los concibe como creencias, o bien como decisiones que han superado un proceso de validación. Los EdP pueden formularse en términos de que la decisión de que un hecho está probado se haga depender de la adopción de una creencia en favor de su existencia (lo que antes era una conjetura pasa a ser una creencia que se estará dispuesto a defender) o bien, de que se le acepte como el resultado de un procedimiento de validación ${ }^{36}$, independientemente que se crea en él (lo que antes era una conjetura pasa a ser un enunciado aceptado).

Las dos opciones recién indicadas se presentan también en la cotidianeidad. Así, respecto de una determinada aserción yo puedo creerla o no creerla, aceptarla o no aceptarla; y luego es de esperar que actúe en consecuencia. Creer algo no me releva de la obligación de dar razón de mis dichos y hacerme cargo de las refutaciones que se hagan; aceptar algo provoca efectos muy parecidos. Hay, sin embargo, una diferencia insoslayable entre ambas opciones al momento de asignarle un papel a la voluntad: no se cree a voluntad, incluso si se reconoce

\footnotetext{
${ }^{36}$ Esto supone una concepción de los hechos como conjeturas que habiendo sido sometidas a un proceso de validación, dejan de ser problematizadas por haberse desvanecido las razones para ello (la última palabra ya fue dicha).
} 
Los usos de los estándares de prueba: entre umbrales y prototipos

que se puede voluntariamente llevar a cabo una serie de acciones que propenden a que yo llegue a creer o a descreer de algo. Cuando, en cambio, acepto una aserción que ha cumplido con ciertas condiciones de validación es porque he tomado una decisión en tal sentido ${ }^{37}$. Las personas, por cierto, tendemos a que lo que creemos y lo que aceptamos sea coincidente, pero estamos dispuestos a hacer excepciones ${ }^{38}$.

Un EdP como el de la PP calza bien con la concepción de los hechos como un acto de aceptación derivado de la concurrencia de distintas variables (cantidad de pruebas, relevancia de las pruebas, etc.), independientemente de cuales sean mis creencias. En casos en que las pruebas disponibles favorables a la versión de la demandante sean solo ligeramente superiores a aquellas que presenta la demandada, será frecuente que más que una creencia, se produzca solo una disposición favorable hacia una creencia ${ }^{39}$ (aunque se esté en condiciones de sostener que probablemente los hechos debatidos ocurrieron, el adjudicador habitualmente dejará en suspenso las creencias que vayan en ese sentido). El MADR, en cambio, al menos en algunas de sus formulaciones ${ }^{40}$, puede hacerse calzar perfectamente con la concepción de hechos probados como dependientes de la producción de una determinada creencia (creencia firme respecto de la cual no se tienen dudas razonables), pasando a segundo plano la superación del proceso de validación

37 Otra diferencia refiere a que cuando se trata de adoptar una creencia, el sujeto tiene como referente su sistema de creencias previas no teniendo que dar razones de aquello, a menos que le sea solicitado (ver Levi, I., "Pragmatism and Change of View", en Levi, I., Pragmatism and Inquiry, Selected Essays, Oxford: Oxford University Press, 2012, p. 30 y ss.). El sujeto considera sus creencias actuales como lo dado y el juicio sobre la potencial nueva creencia es básicamente de consistencia con dicho sistema. En el caso que lo que se pida al sujeto sea la aceptación de la creencia, se le está situando en una situación de indiferencia respecto de su sistema de creencias exigiéndole un mayor compromiso con aquello que culturalmente se acepta.

${ }^{38}$ Ver Cohen, L. J., "Belief and acceptance", Mind, XCVIII, 1989, p. 368; Mosterín, J., Lo mejor posible. Racionalidad y acción humana, Madrid: Alianza, 2008, p. 191 y ss.

${ }^{39} \mathrm{La}$ actitud que mejor representa ese estado de cosas será la de suspensión de creencias ( $\mathrm{es}$ posible que $\mathrm{P}$ haya ocurrido, pero no descarto que no haya ocurrido!).

${ }^{40}$ El art. 340 del Código Procesal Penal chileno dice: "Nadie podrá ser condenado por delito sino cuando el tribunal que lo juzgare adquiriere, más allá de toda duda razonable, la convicción de que realmente se hubiere cometido el hecho punible". 
propiamente tal. También es posible que simultáneamente concurran ambas aproximaciones. Aquello ocurre si para dar superado el MADR se requiere la producción de un estado de creencias (no basta la aceptación) y, en cambio, para entender que no ha sido superado basta con aceptar una duda razonable procedimentalmente validada, incluso si el juzgador persevera en su creencia de la culpabilidad.

\section{II.1. Los macro-usos de los estándares de prueba}

Los usos anunciados pueden agruparse en relación a tres macro-usos esperables de los EdP. Entenderé por macro-uso un conjunto de usos que presentan características comunes relevantes.

i) Determinación de la cantidad de errores esperables del sistema de adjudicación. Si se tienen en cuenta los usos de las letras a), b) y c), cabe sostener que un EdP exigente favorece decisiones más fiables que las esperables de la utilización de un EdP menos exigente. Aquello ocurre en razón de que con un EdP exigente, la parte demandante o acusadora elige solo «buenos casos»; es decir, aquellos en los que vislumbra posibilidades de éxito. Dado que en ellos se debe producir un mayor número de pruebas y argumentos de partes, los jueces están en mejor posición para evitar errores al momento de tomar la decisión ${ }^{41}$. El asunto no termina ahí, pues esa clase de EdP propende a una reducción de la magnitud de los saltos argumentales en el texto justificatorio. De esta manera, los EdP no tienen un uso que solo se relaciona con la distribución de errores, sino también con la cantidad de errores tolerables.

Las investigaciones que llevamos a cabo en diferentes contextos —incluido el de los procesos judiciales - propenden, por una parte, a aumentar los conocimientos; y por la otra, a reducir los errores, tanto

${ }^{41}$ Ver Keynes, J. M., A Treatise of Probability, London: Macmillan and Co., 1921, pp. 78-79. Lo expresado explica una aparente paradoja que se produce en cuanto a que en juicios orales penales (en que se opera con el MADR), la proporción de casos en que se dan por probados los hechos suele ser más alta que en aquellos que se aplica la PP (los juicios civiles). No hay tal paradoja pues en los juicios penales orales hay filtros más exigentes que evitan el ingreso de casos con prueba escasa, a la vez que el esfuerzo por optimizar la prueba es mucho mayor. 
Los usos de los estándares de prueba: entre umbrales y prototipos

en los que consideramos como nuestros saberes actuales, como en los potenciales $^{42}$. Un EdP exigente afecta las pretensiones cuantitativas (habrá menor cantidad de hechos probados) pero es deferente con las cualitativas (se reconocerá que se saben menos cosas, pero serán más seguras $)^{43}$. Al contrario, un EdP menos exigente aumenta los saberes, pero se verán amenazados por un mayor número de errores.

ii) Distribución entre las partes de los errores que produce el sistema de adjudicación. Esta categoría está conformada por los usos indicados en las letras d) y e). Según lo ya señalado, al menos en el estado actual de desarrollo, entender los EdP como herramientas que permiten distribuir los riesgos de falsos positivos y de falsos negativos - entendidos en sus términos fuertes - constituye una meta que excede las posibilidades de los EdP. Una opción más viable pasa por entender los EdP, ya sea como mecanismos que fijan un umbral de resistencia a la falsación ${ }^{44}$, o bien como prototipos discursivos que determinan cuanto soporte requiere el relato de la parte sobre la que recae la carga de persuasión.

El MADR es un EdP exigente pues implica la exclusión de cualquier duda razonable en las cadenas argumentales ${ }^{45}$ que dan sustento a la acusación. Con ello se espera una menor proporción de falsos positivos que de falsos negativos, bajo la condición de que las partes

${ }^{42}$ Ver Levi, I., The fixation of belief and its undoing. Changing beliefs through inquiry, Cambridge: Cambridge University Press, 2009, pp. 10-11.

${ }^{43}$ Lo expresado se produce en la medida en que la negación que acompaña al EdP sea ilocucionaria y no proposicional.

${ }^{44}$ Los esfuerzos de los tribunales de justicia por ocultar en sus sentencias condenatorias los espacios de incertidumbre que han dado por superados - pese a las lagunas de información que afecten al caso concreto - no constituye una estrategia que favorezca un genuino uso de los EdP. Mucho más interesante es la explicitación de las razones de por qué pese a esos fallos de información se decide adecuadamente dando los hechos por probados. Ver, en un sentido acorde al indicado, Gardner-Medwin, T., "Reasonable doubt: Uncertainty in Education, Science and Law", en Dawid, Ph., Twining, W. y Vasilaki, M. (eds.), Evidence, Inference and Enquiry, Oxford: The British Academy, 2011, p. 466 y ss.

${ }^{45}$ Si se trata de argumentos coordinados (interdependientes), una duda razonable en cualquiera de las líneas de argumentación será suficiente para derribar los hechos afirmados por la parte acusadora. Si, en cambio, los argumentos son múltiples (independientes) habrá que analizar la red argumental completa para llegar a sostener que la duda razonable es un impedimento para dar por probados los hechos. 
se encuentren en una posición simétrica en lo que concierne a las posibilidades de producir prueba o de persuadir (o bien, cuando habiendo asimetría no afecta al acusado $)^{46}$. Con un EdP menos exigente como la PP no se pretende, en cambio, provocar desequilibrios relevantes entre falsos positivos y falsos negativos, bajo condición de que las partes se encuentren en una posición simétrica.

Las opciones que se hacen competir conforme a lo planteado en la letra e) inciden en la distribución de los riesgos de error. Al operar en un sistema en que la negativa a dar por probado obedece a una suspensión del juicio (negación ilocucionaria) los falsos negativos aumentan proporcionalmente; en cambio si se opera en un sistema en que la negativa a dar por probado los hechos implica aceptar la conducta opuesta a la que se trataba de demostrar (está probado no X), entonces disminuye la proporción de falsos negativos.

iii) Forma que adoptan los hechos probados. Este macro-uso agrupa los usos ya indicados en las letras f) y g). Respecto de la letra g), los EdP dan espacio a concepciones en que las creencias de quienes se desempeñan en el rol de jueces son gravitantes; como también a aquellas en que lo relevante es la aceptación de una conjetura en consideración de la fortaleza de las conexiones establecidas respecto de las pruebas disponibles. Lo expresado no es obstáculo a que en el primero de los casos resultará muy difícil operar con estándares de revisión de los hechos que se han dado o no por probados, dado que lo que mediante ellos habría que poner en duda es la sinceridad de los adjudicadores. En el segundo de los casos, un tribunal superior no debiese tener dificultades —más allá de las propias de definir los alcances del estándar de revisión y del conocimiento del caso concreto- para llegar a modificar lo que fue resuelto. Sin perjuicio de lo señalado, en el primero de los casos conserva sentido sostener que los tribunales hacen depender su decisión de lo que estipulan los $\mathrm{EdP}^{47}$.

46 Ver Posner, R., "An Economic Approach to the Law of Evidence", Stanford Law Review, Vol. 51, 1998-1999, p. 1505.

47 En los casos en que resulte absurdo afirmar que se ha alcanzado una cierta creencia, el sistema jurídico y social cuentan con resguardos, tales como sanciones disciplinarias, crítica abierta a las decisiones, etc. 
Los usos de los estándares de prueba: entre umbrales y prototipos

Los EdP pueden ser usados en términos de privilegiar (o no) hechos consistentes con lo que se encuentra aceptado en espacios de discusión que excedan lo puramente jurídico. Si a los jueces se les pide aceptar hechos que puedan también usarse fuera del espacio del discurso de lo jurídico, es más fácil lograrlo si se recurre a una forma de razonamiento holística.

En rigor, varios de los usos de los EdP se vinculan a la inexistencia de disposiciones que aluden a lo que aquellos regulan. En ese sentido, tales usos son contingentes. Así, por ejemplo, los usos b), e), f) y g) podrían independizarse de los EdP si se introdujesen disposiciones que los traten directamente. De aquello resultaría que se restringirían los usos de los EdP a los más ineludibles, como sería el caso de d) y, en menor medida, de a) y c).

\section{Un ejercicio de toma de decisiones con EdP}

El desencanto aludido al comienzo de este trabajo se relaciona estrechamente con el fracaso en la reducción de los resultados del análisis probatorio a un guarismo ( la conjetura $\mathrm{X}$ ha sido probada un 0,7 dentro de un rango de 0 a 1») o, al menos, en la construcción de textos justificantes de la decisión cuyos contornos sean muy precisos («la conjetura $\mathrm{X}$ ha sido sólidamente confirmada», donde «sólidamente confirmada» se diferencia de manera nítida de «medianamente confirmada» o de «levemente refutada»). Los problemas inherentes a las metodologías utilizadas y/o a las precariedades de la cultura jurídica para aplicar el cálculo probabilístico han redundado en que el uso de números no pase de ser una metáfora ${ }^{48}$, salvo, tal vez, unos pocos casos que efectivamente han resultado provechosos ${ }^{49}$.

${ }^{48}$ La dificultad para limar los contornos de los conceptos hace difícil no establecer relaciones con números si se quiere comunicar precisión. Gascón, M., "Sobre la posibilidad de formular estándares de prueba objetivos", Doxa, 28, 2005, p. 135 y ss.

${ }^{49}$ Los intentos más evidentes en ese sentido han apuntado a la aplicación del Teorema de Bayes a los asuntos probatorios. Hay una literatura abundante tanto a favor como en contra. Hasta la fecha, hay problemas no resueltos en el cálculo probabilístico, en cuanto herramienta útil para la gestión de los problemas probatorios. Ver, entre otros, 
A continuación, presentaré una secuencia de pasos que toma en cuenta los usos de los EdP previamente señalados. Las posibilidades de ordenación sugeridas pueden variar, como también se pueden añadir o eliminar pasos. Algunas de las formulaciones de los pasos se explican desde una comprensión de los EdP preferente comprometida con su dimensión de umbral y otras, con la de prototipos. En la columna de la izquierda se tendrán especialmente presentes algunas de las posibilidades que ofrece la $\mathrm{PP}$ y en la derecha, algunas lecturas posibles de lo que implica el MADR.

Fase 1. Determine cuál es la opción opuesta a la de «probada la conducta X». Luego de ello, siga los pasos señalados bajo la respectiva columna.

Probada la conducta no $X$

i) Identifique las piezas de prueba (y argumentos) que fortalezcan la conjetura $\mathrm{X}$.

ii) Identifique las piezas de prueba (y argumentos) que debiliten la conjetura $\mathrm{X}$.

iii) Identifique las piezas de prueba (y argumentos) que fortalezcan la conjetura no $\mathrm{X}$.

iv) Identifique las piezas de prueba (y argumentos) que debiliten la conjetura no $\mathrm{X}$.
No probada la conducta $X$

i) Identifique las piezas de prueba (y argumentos) que fortalezcan la conjetura $\mathrm{X}$.

ii) Identifique las piezas de prueba (y argumentos) que debiliten la conjetura $\mathrm{X}$.

iii) Identifique los argumentos que resulten consistentes con la suspensión de toma de posición respecto de la conjetura $\mathrm{X}^{*}$.

* Esta situación puede darse en el caso de desacuerdo entre los juzgadores respecto de si la conjetura $\mathrm{X}$ ha sido probada (o no) a partir de la prueba disponible. La sola existencia de un desacuerdo constituye una razón para ser más cautos en la decisión, aun cuando no imposibilite considerar probada la conjetura X. Ver Coloma, R., "Dos es más que uno, pero menos que tres. El voto disidente en decisiones judiciales sometidas al estándar de prueba de la 'duda razonable"', Política criminal, 9 (18), 2014, pp. 400-427.

Haack, S., "El probabilismo jurídico: una disensión epistemológica", en Vásquez, C., (ed.), Estándares de prueba y prueba científica. Ensayos de epistemología jurídica, Madrid: 
Los usos de los estándares de prueba: entre umbrales y prototipos

Fase 2. Identifique la estructura de razonamiento preferente para conectar la prueba disponible y la conjetura sometida a contrastación. Luego siga los pasos señalados bajo la respectiva columna.

Razonamiento holístico

\section{Razonamiento atomista}

i) Identifique distintos relatos posibles que articulen el material probatorio disponible en términos de fortalecer la conjetura a probar (X).

ii) Elija aquella opción que se muestre más útil para la validación de la conjetura $X$.

iii) Identifique distintos relatos posibles que articulen el material probatorio disponible en términos de fortalecer la conjetura a probar (no $X^{*}$ ).

iv) Elija aquella opción que se muestre más útil para la demostración de la conjetura no $\mathrm{X}$. i) Desglose la conjetura a ser probada $\mathrm{X}$ en distintas proposiciones interdependientes que, a su vez, requieran ser demostradas (penúltima probanda) ${ }^{* *}$.

ii) Establezca conexiones entre la penúltima probanda y la prueba disponible que ha seleccionado en la fase previa.

iii) Identifique en las distintas cadenas de argumentos, las partes que resultan más débiles.

* Se operará de esta manera si el opuesto a «probado X» fuere «probado no X».

** Ver Anderson, T., Schum, D., Twining, W., Análisis de la prueba, Madrid: Marcial Pons, 2015, p. 409. 
Fase 3. Determine si los hechos probados deben entenderse como creencias o como conjeturas que cuentan con credenciales para ser aceptadas. Luego siga los pasos que se indican más abajo.

Hechos probados como creencias

i) Realice un ejercicio de introspección acerca de la información relevante producida en la audiencia de prueba que le parezca digna de ser tomada en cuenta (a su juicio, es prueba fiable).

ii) Realice un ejercicio de introspección acerca de qué argumentos que toman en cuenta la información que Ud. ha considerado fiable establecen conexiones con las conjeturas rivales en términos de fortalecerlas o de debilitarlas.

iii) Analice los resultados obtenidos de los pasos i) y ii) desde la perspectiva de si resistirán el escrutinio del auditorio al que se dirige la sentencia*.
Hechos probados como conjeturas con credenciales para su aceptación

i) Analice - desde la perspectiva de una comunidad racionallas piezas de información producida en la audiencia de prueba que sean aptas para soportar una decisión que da por probada la conjetura $\mathbf{X}$.

ii.) Analice - desde la perspectiva de una comunidad racional- la fiabilidad de las pruebas rendidas.

iii.) Analice -desde la perspectiva de una comunidad racionallas fortalezas y debilidades de posibles conexiones entre las pruebas disponibles y la conjetura X.

* Este paso tiene en cuenta que la decisión debe ser motivada en un texto. 
Los usos de los estándares de prueba: entre umbrales y prototipos

Fase 4. Evalúe la suficiencia de la prueba y de los argumentos que ha identificado en las fases previas, a los efectos de construir un relato. Tenga en consideración tanto si el relato es más fuerte que el alternativo, o bien si es apto para superar la inercia de lo no probado.

\begin{tabular}{cl}
\hline $\begin{array}{c}\text { Fortaleza de relatos consistentes con } \\
\text { "probado } X » y \text { los de "probado no } X "\end{array}$ & \begin{tabular}{l} 
nos de superar la inercia de lo no probado \\
\hline
\end{tabular} \\
\hline
\end{tabular}

Evalúe lo siguiente, tanto respecto de «probado $\mathrm{X}$ » como de «probado no $\mathrm{X}$ »:

i) Relevancia del relato (conexión suficiente con las normas abstractas que pretenden aplicarse); ii) Coherencia de la narración (compatibilidad entre sus distintas partes); iii) Completitud o cobertura del relato (magnitud de las lagunas de información habida cuenta del contexto ¿podrían haber sido menos?, ¿quién es responsable de que se hayan producido?, etc.); iv) Anclaje con un background de conocimientos prestigiosos; v) Anclaje con la información producida en la audiencia de prueba (extensión de los saltos inferenciales que se realizan para los efectos de hacer aparecer el relato como una derivación de las que han sido válidamente presentadas como pruebas)*.

Pregunta clave: ¿Cuál de los relatos es más satisfactorio respecto de las variables identificadas?
Evalúe lo siguiente respecto de «probado X» (realice los mismos pasos de la columna de la derecha)

Pregunta clave: ¿Es el relato suficiente como para superar la inercia que favorece al punto de vista de lo que no se encuentra probado?

* Coloma, R., "Las buenas y las malas historias. Criterios de validación del discurso de los hechos en las sentencias judiciales", en Brunet, P. y Arena, F. (dirs.), Cuestiones contemporáneas de teoría analitica del derecho, Madrid: Marcial Pons, 2011, p. 87 y ss. 
Fase 5. Decida de una manera deferente con las mayores o menores exigencias asociadas al EdP ${ }^{50}$.

En esta última fase no se distinguen dos columnas, pese a que hay efectos diversos según sea el EdP que se aplique. Se asumen los límites de los seres humanos para distinguir con un cierto nivel de precisión los niveles de logro de la actividad probatoria. Bajo una comprensión de los EdP que resulta más cercana a la de umbral que a la de prototipo, Kevin Clermont ha propuesto distinguir siete niveles de demostración de los hechos: los extremos están conformados por los enunciados existe la más leve posibilidad, hasta es casi cierto $^{51}$. Operar bajo ese supuesto provee una adecuada dosis de realismo ${ }^{52}$. Hay dificultades evidentes para comparar casos con diferentes soportes probatorios, salvo aquellos en que se persigan responsabilidades equiparables y se cuente con pruebas similares.

Ante un caso cualquiera, el tribunal que decide los hechos se pregunta si las pruebas y las argumentaciones disponibles sitúan (o no) el caso en la zona de penumbra de la locución «hecho probado». Si tanto las pruebas como los argumentos resultan apabullantes en favor de que la conjetura ha de darse por probada (zona de claridad) —o bien, si pruebas y argumentos son ínfimos como para tomar en serio la opción de que los hechos están probados (zona de obscuridad)— no será nece-

${ }^{50}$ Esta fase puede realizarse: i) además de la fase 4; ii) en lugar de la fase 4; o bien iii) omitirse si la fase 4 ha sido suficientemente satisfactoria y la concepción de EdP que se utiliza es la de prototipos.

${ }^{51}$ Clermont, K., op. cit., p. 35 y ss. Con los siete niveles habrá tres sobre un estado de equilibrio y tres bajo aquel.

${ }^{52}$ Los médicos, con frecuencia, preguntan a los pacientes acerca de la intensidad de un dolor, pese a que no les resulta posible conocer de antemano su tolerancia al dolor (y eso puede variar en cada paciente). Usan, generalmente, una escala 1 a 10 y dan algunas pistas de lo que implica situarse en uno de los extremos ("1 es un dolor que si bien Ud. advierte resulta fácilmente soportable, no provocándole mayores problemas para seguir ejecutando sus labores cotidianas; 10 es un dolor extremo que Ud. considera que no podrá seguir soportando o con el que Ud. prefiere no seguir viviendo"). Los resultados que obtienen les permiten evaluar la progresión de este síntoma en un mismo paciente y así tomar decisiones acerca de los cursos a seguir. 
sario siquiera plantearse el problema de la utilización de un $\mathrm{EdP}^{53}$. Si, en cambio, tanto las pruebas como los argumentos disponibles llevan al adjudicador a situar el caso concreto en la zona de penumbra de la locución «hechos probados», el tribunal debe tomar posición respecto a cuál de las siguientes tres categorías representa mejor el caso concreto:

\begin{tabular}{lll}
\hline $\begin{array}{l}\text { Conjetura escasamente } \\
\text { demostrada }\end{array}$ & $\begin{array}{l}\text { Conjetura medianamente } \\
\text { demostrada }\end{array}$ & $\begin{array}{l}\text { Conjetura altamente } \\
\text { demostrada* }\end{array}$ \\
\hline
\end{tabular}

* Operar con tres niveles se asemeja a la elección de prendas de vestir: S/M/L. A ellas se van agregando XS, XL.

Luego de tomar posición, será preciso determinar si el caso queda mejor situado, ya sea en la parte inferior o bien en la superior de la categoría elegida.

\begin{tabular}{|c|c|c|}
\hline $\begin{array}{l}\text { Conjetura escasamente } \\
\text { demostrada }\end{array}$ & $\begin{array}{l}\text { Conjetura medianamente } \\
\text { demostrada }\end{array}$ & $\begin{array}{l}\text { Conjetura altamente } \\
\text { demostrada }\end{array}$ \\
\hline+ & + & + \\
\hline
\end{tabular}

Una vez que se ha situado el caso en una de las seis posiciones hay que considerar un EdP en concreto. Por ejemplo, si se trata del MADR se exige operar en el umbral Alto (-) o Alto (+). A su vez desde la perspectiva de la refutación se exigiría no operar en una categoría superior a escasa $(+)$, ya que en caso contrario habría una duda razonable. Así, la combinación confirmación Alta (-) y refutación baja (+) constituye el mínimo requerido para superar el MADR. Respecto de la $\mathrm{PP}$, en principio, la decisión se tomará caso a caso, pues dependerá de la posición que ocupe «probado $\mathrm{X}$ » $\mathrm{v} / \mathrm{s}$ «no probado $\mathrm{X}$ ». La posición vencedora será la que alcance la posición más alta, con la salvedad que — según se ha dicho — en la práctica a la posición «probado X» se le 
exige un mínimo (no relacional) para que sea seriamente considerada: por ejemplo, conjetura medianamente demostrada (-).

Lo recién expresado se puede ajustar, sin grandes dificultades, a la concepción de los EdP como prototipos teniéndose en consideración la cercanía o lejanía respecto del prototipo que en concreto se defina. Un EdP exigente requerirá que en el caso concreto haya un alto nivel de aproximación al prototipo, en cambio, uno escasamente exigente hace posible una mayor distancia.

\section{Conclusiones}

Los EdP son una clase de directivas de las cuales se espera que cumplan un papel clave en la configuración del razonamiento probatorio. Se sospecha - sin embargo- que aquellas expectativas no han sido, hasta ahora, suficientemente logradas. La influencia aparentemente escasa que ejercen los EdP en la toma de decisiones probatorias se debería - en una importante medida - a que la labor teórica no ha logrado clarificar algunos aspectos relevantes de su uso.

En este texto se ha sostenido que los estándares pueden reconstruirse ya sea como umbrales o como prototipos. El asunto no es menor ya que dependiendo de si se entienden en uno $u$ otro sentido, las operaciones que deberán llevarse a cabo con los EdP serán diferentes. Concebir los EdP como umbrales privilegia formas de análisis de carácter cuantitativo. Estas suelen provocar bastantes complicaciones a los juristas, ya que - al menos por el momento - no ha podido resolverse el problema de cómo realizar mediciones. Concebir a los EdP como prototipos privilegia, en cambio, aproximaciones de carácter cualitativo. Aquellas se traducen en una evaluación de las conjeturas problematizadas desde la perspectiva de sus semejanzas y diferencias respecto a uno o más casos indubitados: habrá así un límite de que las comparaciones resultantes serán solo ordinales.

Además de la distinción conceptual precedente, se han hecho visibles distintos usos de los EdP. De esta manera, se refuerza la idea de 
que si bien la distribución de los errores constituye un uso importante (aunque dudoso) de los EdP, no sería el único. Los EdP son los responsables de una serie de decisiones que adoptan las partes y los jueces en el decurso de los procesos judiciales. Así —a fin de cuentas- el principal problema de los EdP no sería su falta de operatividad, al menos en el sentido fuerte de la locución.

\section{Bibliografía}

Allen, R., "Los estándares de prueba y los límites del análisis jurídico", en Vásquez, Carmen (ed.), Estándares de prueba y prueba cientifica. Ensayos de epistemología jurídica, Madrid: Marcial Pons, 2013.

Amaya, A., "Diez tesis acerca de la coherencia en el derecho" Discusiones X, 2011, pp. 21-64.

Anderson, T., Schum, D. y Twining, W., Análisis de la prueba, Madrid: Marcial Pons, 2015.

Arnau, J., Arte de probar. Ironía y lógica en India Antigua, Madrid: FCE, 2008.

Austin, J. L., "Otras mentes", en Urmson, J. O. y Warnock, G. J. (coomp.), Ensayos filosóficos, Madrid: Alianza, 1989.

Bayón, J. C., "Epistemología, moral y prueba de los hechos: hacia un enfoque no benthamiano", Analisi e Diritto, 2008.

Clermont, K., Standards of Decision in Law, Durham: Carolina Academic Press, 2013.

Cohen, L. J., "Belief and acceptance", Mind, XCVIII, 1989.

Coloma, R., "Dos es más que uno, pero menos que tres. El voto disidente en decisiones judiciales sometidas al estándar de prueba de la «duda razonable»", Politica criminal, 9 (18), 2014, pp. 400-427.

Coloma, R., "Estándares de prueba y juicios por violaciones a los derechos humanos", Revista de Derecho (Valdivia), XXII (2), 2009, pp. 205-229.

Coloma, R., "Las buenas y las malas historias. Criterios de validación del discurso de los hechos en las sentencias judiciales", en Brunet, 
P. y Arena, F. (dirs.), Cuestiones contemporáneas de teoría analítica del derecho, Madrid: Marcial Pons, 2011.

Coloma, R. y Agüero, C., "Lógica, ciencia y experiencia en la valoración de la prueba", Revista Chilena de Derecho, 41 (2), 2014, pp. 673-703.

Dei Vecchi, D., "Tres discusiones acerca de la relación entre prueba y verdad", Discusiones, XIII, 2013, pp. 233-264.

Elster, J., Juicios salomónicos. Las limitaciones de la racionalidad como principio de decisión, Barcelona: Gedisa, 1991.

Ferrer, J., La valoración racional de la prueba, Madrid: Marcial Pons, 2007.

Gardner-Medwin, T., "Reasonable doubt: Uncertainty in Education, Science and Law", en Dawid, Ph., Twining, W. y Vasilaki, M. (eds.), Evidence, Inference and Enquiry, Oxford: The British Academy, 2011.

Gascón, M., "Sobre la posibilidad de formular estándares de prueba objetivos", Doxa 28, 2005.

Haack, S., "El probabilismo jurídico: una disensión epistemológica", en Vásquez, C. (ed.), Estándares de prueba y prueba científica. Ensayos de epistemología jurídica, Madrid: Marcial Pons, 2013.

Hart, H. L. A., El concepto de derecho, Buenos Aires: Abeledo-Perrot, 1995 (segunda edición).

Ho, H. L., A Philosophy of Evidence Law, Oxford: Oxford University Press, 2010 (reimpresión).

Keynes, J. M., A Treatise of Probability, London: Macmillan and Co., 1921.

Larroucau, J., "Hacia un estándar de prueba civil", Revista Chilena de Derecho, 39 (3), pp. 783-808.

Laudan, L., "Una breve réplica", Doxa, 28, 2005.

Laudan, L., "Por qué un estándar de prueba subjetivo y ambiguo no es un estándar", Doxa, 28, 2005.

Laudan, L., Verdad, error y proceso penal, Madrid: Marcial Pons, 2013.

Levi, I., "Pragmatism and Change of View", en Levi, I., Pragmatism and Inquiry, Selected Essays, Oxford: Oxford University Press, 2012. Levi, I., The fixation of belief and its undoing. Changing beliefs through inquiry, Cambridge: Cambridge University Press, 2009. 
Mosterín, J., Lo mejor posible. Racionalidad y acción humana, Madrid: Alianza, 2008.

Nozick, R., The nature of the rationality, tercera edición, Princeton: Princeton University Press, 1995.

Páez, A., "Estándares múltiples de prueba en medicina y derecho", en Páez, A. (coord.), Hechos, evidencia y estándares de prueba, Bogotá: Universidad de los Andes, 2015.

Pepper, S., World Hypotheses, Berkeley: University of California Press (impresión según demanda), 1942.

Posner, R., "An Economic Approach to the Law of Evidence", Stanford Law Review, 51, 1998-1999.

Schauer, F., Profiles, Probabilities and Stereotypes, Cambridge \& London: The Belknap Press of Harvard University Press, 2006.

Taruffo, M., "Tres observaciones sobre 'Por qué un estándar de prueba subjetivo y ambiguo no es un estándar' de Larry Laudan", Doxa, 28, 2005.

Toulmin, S., Los usos de la argumentación, Barcelona: Península.

Whitman, J., The origins of reasonable doubt. Theological roots of the criminal trial, New, Haven \& London: Yale University Press, 2008.

Williams, B. y Nagel, Th., La suerte moral, Oviedo: KRK, 2013.

Zadeh, L., "Fuzzy logic and approximate reasoning", Synthese 30, 1975. 